\title{
Globe
}

Revue internationale d'études québécoises

\section{Penser la religion au Québec}

\section{Raymond Lemieux}

Volume 11, numéro 1, 2008

URI : https://id.erudit.org/iderudit/1000500ar

DOI : https://doi.org/10.7202/1000500ar

Aller au sommaire du numéro

Éditeur(s)

Globe, Revue internationale d'études québécoises

\section{ISSN}

1481-5869 (imprimé)

1923-8231 (numérique)

Découvrir la revue

Citer cet article

Lemieux, R. (2008). Penser la religion au Québec. Globe, 11(1), 225-236.

https://doi.org/10.7202/1000500ar d'utilisation que vous pouvez consulter en ligne.

https://apropos.erudit.org/fr/usagers/politique-dutilisation/ 


\section{PERSPECTIVE}

\section{PENSER LA RELIGION \\ AU QUÉBEC}

RAYMOND LEMIEUX

Université Laval

$+4++4+4+4++4+4+4++++4+4+$

Ils ne mouraient pas tous, mais tous étaient frappés.

Jean de LA FONTAINE,

"Les animaux malades de la peste"

Les débats récents montrent la difficulté d'établir un diagnostic contemporain sur la question religieuse au Québec. Et en même temps, ils font resurgir l'importance paradoxale de cette question qu'on aurait pu croire dépassée. Que signifient ces résurgences d'intérêt pour le religieux et ces débats médiatiques prenant parfois des accents dramatiques?

On fait généralement remonter l'histoire de la sécularisation du Québec à la Révolution tranquille. Celle-ci a représenté l'affirmation d'une modernité, non pas brusquement découverte comme beaucoup se sont plu à le proclamer, mais déjà à l'œuvre par l'urbanisation, la mobilité des populations migrant vers les marchés du travail, la transformation des paysans en ouvriers et employés, de même que le développement des petites et moyennes entreprises hors tutelle de la bourgeoisie coloniale. La Révolution tranquille a imposé les lumières des classes moyennes scolarisées à l'obscurité des raisons d'autorité jusque-là mises en avant par les élites clérico-conservatrices. 
La machinerie lourde de la modernité a alors arasé les chemins difficiles de la tradition pour en faire des autoroutes vers le progrès. Au tournant du XXI' siècle, ce Québec qu'on accuse encore d'avoir été sous tutelle cléricale et incapable, à cause de cela, d'un véritable développement, est devenu l'une des sociétés les plus radicalement sécularisées de l'Occident. Quoiqu'on possède relativement peu de données sûres à ce propos, celles dont on dispose sont parfois surprenantes. Dans un sondage effectué en 2002 auprès de jeunes Canadiens de toutes confessions et de toutes les provinces, les répondants québécois, dont $72 \%$ disaient "s'identifier à la tradition catholique", $y$ ont régulièrement montré des taux d'adhésion à cette tradition inférieurs à ceux de toutes les autres catégories. Les Québécois francophones, en particulier, y présentent des attitudes plus critiques et plus distanciées envers le catholicisme que tous les autres Canadiens, y compris les catholiques québécois allophones, les catholiques canadiens-français des autres provinces et les non-catholiques de toute région et de toute langue! $\mathrm{Ce}$ paradoxe devient même assez bizarre quand on voit des répondants protestants anglophones, par exemple, se dire davantage d'accord avec certains énoncés romains de la morale catholique que les catholiques québécois francophones'.

On peut donc conclure sans grand risque à la rupture des nouvelles générations par rapport à leurs supposées racines religieuses. Si les statistiques sont insuffisantes pour le démontrer, les blessures de la mémoire en témoignent bien, de même que l'oubli généralisé des données de cette culture religieuse, voire l'acharnement de certains à en attaquer les dépouilles.

Mais tout un ensemble de questions se télescopent quand on commence à tenir compte de la "différence " québécoise, sur le plan religieux. Tout d'abord, que signifie, concrètement, cette sécularisation rapide et radicale et quelle en est la portée réelle? Si le Québec francophone est devenu une société postcatholique, à vrai dire l'une des premières ${ }^{2}$ en contexte de globalisation, l'effondrement de ses cadres traditionnels ne se solde-t-il pas par d'autres modes d'encadrement, répondant d'une autre logique, mais à portée contraignante comparable? Comment dès lors

$$
+4
$$

1. On peut trouver un résumé de ces données fournıes par Radio-Canada, qua avatt commandé le sondage a l'occasion des Journées mondiales de la jeunesse de Toronto, dans notre artıcle *Catholıcisme et fonction identitaire: du "Canada français" au Québec contemporain ", G1lles GAGNE [dir.], Le Canada français. Son temps, sa nature, son héritage. Les sémınatres Fernand-Dumont, Québec, Nota bene, 2006, p. 29-43.

2. Mais sans doute pas la dernière, si l'on consıdère les transformations contemporaines de l'Irlande, de l'Espagne et de la Pologne, entre autres. 
qualifier cette dernière dans ses rapports à la culture et à la vie publique? Jusqu'à quel point le nous québécois est-il encore pétri de farine religieuse? Et de quoi parle-t-on effectivement quand on évoque la "religion"? Des seules institutions, traditionnelles ou nouvelles, endogènes ou exogènes, qui sont ainsi qualifiées? Des croyances, des représentations capables de donner cohésion aux pratiques sociales, des visions du monde qui président, pour chacun, au bricolage d'un sens à sa vie? Où gîte désormais le sacré - cet intouchable, séparé, attirant et redoutable - dans les sociétés sécularisées comme la québécoise?

Jusqu'à quel point les signes religieux qui s'affirment sur la place publique sont-ils des rappels du sacré, représentant des poussées militantes susceptibles de menacer la laïcité des institutions? Ne sont-ils pas plutôt des manifestations mineures - ailleurs, on pourrait sans doute parler d'avatars d'institutions religieuses sans emprise efficace sur la vie publique parce qu'elles ont perdu, ou n'ont jamais eu, la capacité de contrôler la culture? On excusera la comparaison facétieuse, mais ne sont-ils pas comme ces casquettes et chandails à l'effigie des champions sportifs, portés par des admirateurs qui s'identifient ainsi à ces vedettes, mais ne pratiqueront jamais les sports en question? Quelle distance y a-t-il entre l'affichage du signe religieux et l'intégration, dans la vie quotidienne, de la religiosité qu'il représente? Certes, on ne peut mettre sur le même plan, de ce point de vue, le foulard (dit «islamique») et la croix portée en sautoir sur un décolleté mondain, ni même le voile de certaines religieuses et le kirpan. Chacun de ces signes renvoie à l'histoire personnelle et (ou) communautaire de celui qui l'affiche. Dans une culture éclatée, la portée réelle des signes religieux est d'ailleurs souvent bien plus profane que religieuse. Sinon, quel sort faudrait-il faire aux jurons québécois si allégrement nourris de vocabulaire catholique? Bref, toute une sémiologie ${ }^{3}$ des signes religieux reste à faire. Quels messages portent-ils, explicitement ou implicitement? Pourquoi, malgré leur caractère mineur, certains provoquent-ils de l'urticaire, alors que d'autres restent anodins?

Il est hors de propos de répondre ici à toutes ces questions. Elles explicitent cependant pour nous l'importance, et peut-être l'urgence, de penser le religieux. Qu'entendons-nous par là? Penser le religieux, c'est apprendre à le concevoir non seulement comme un sentiment, une affection

$$
+\div
$$

3. Ferdinand DE SAUSSURE concevaic la sémıologie comme ala science qui étudie la vie des signes au sein de la vie sociale" (Cours de linguistique géntrale, Paris, Payot, 1922, p. 33). Sigmund Freud proposera d'y chercher un a message" qui n'est pas apparent dans le texte lui-même, mass qui reste en quelque sorte chiffré. 
ou une affliction, mais aussi comme une réalité humaine objective, une dimension de la nature humaine qui, comme chacun le sait, se donne à travers la diversité des cultures. Une dimension qui, à la manière de toute autre réalité, présente ses fécondités et ses limites. Or, dans les efforts pour élaborer cette pensée, on rencontre un certain nombre d'impasses, et cela non seulement dans les franges de la société, comme les minorités sectaires, intégristes ou fondamentalistes, mais également dans le monde pluraliste, hypersécularisé et laïc des Québécois. Nous évoquerons ici deux défis qui en découlent.

\section{PREMIER DÉFI : ASSUMEP LE PLURALISME}

Il est une caractéristique du religieux que l'on oublie parfois: tous, sans exception, se sentent compétents à son égard. Ce fait peut sembler banal, certes, et loin de nous l'idée de disqualifier quiconque en le signalant. Au contraire, c'est là une réalité parfaitement normale et légitime, tout simplement parce que l'idée que nous nous faisons du religieux émane toujours, de quelque façon, de l'expérience que nous en avons. Or, qui pourrait dire qu'une expérience est illégitime? La première caractéristique de l'expérience, en effet, est qu'elle s'impose. On l'a ou on ne l'a pas. Immédiate et totale, elle transforme le sujet. Elle se tient comme un temps privilégié dans son histoire personnelle, représentant l' "authentique" capable de discriminer le factice, et devient pour chacun, un trésor intime - souvent indicible - d'où s'arbitre le reste de sa vie.

Tout être humain, sans exception, possède une expérience qui lui fait attester de certaines croyances et contester les autres. Cette expérience peut lui avoir été transmise par tradition ou s'imposer comme une révélation nouvelle, peu importe. Du fait même d'exister parmi d'autres, de participer à la cohérence culturelle d'un groupe humain, chacun peut prétendre à un savoir concernant ce qui définit la cohérence de son être au monde et la cohérence de ce groupe par rapport au chaos extérieur. Qui plus est, dans la mesure où il lui permet de vivre, ce savoir lui semble d'emblée justifié. "Une société ne crée une religion, écrivait le sociologue durkheimien Henri Desroche, que dans la mesure où l'expérience religieuse lui permet de se créer elle-même", et les formes élémentaires de la religion - la religiosité - se structurent du fait que "la société s'atteste comme société dans l'acte même où le sacré se différencie comme sacré ${ }^{4} »$.

$$
+4
$$

4. Henri Desroche, Sociologres religreuses, Paris, Presses universitaires de France, 1968, p. 61. 
On rétorquera que bien d'autres domaines de la vie sociale sont objets de savoirs expérientiels. Tout être humain vivant en démocratie, par exemple, est réputé posséder une expérience et un savoir politiques. C'est même sur cette base qu'est institutionnalisé le suffrage universel. Mais dès que le débat se corse un peu dans la sphère politique, sont convoquées des autorités qui peuvent prétendre tenir leur savoir de sources objectives et vérifiables, et non seulement de leur expérience intime. De la même façon, si je discute de ponts et chaussées, la compétence à laquelle je puis prétendre ne concerne pas ma seule expérience - elle ne me distingue aucunement de tous les autres usagers -, mais, éventuellement, la documentation objective, les études théoriques et pratiques disponibles, bref un savoir institué et, surtout, vérifié. Or, si on peut mettre en doute un savoir objectif pour vice de construction, si on peut infirmer une expérimentation en lui opposant d'autres expérimentations, on ne peut jamais contester celui qui dit: "c'est vrai, j'en ai fait l'expérience", sauf à prétendre qu'il ment ou que ses perceptions sont illusoires, ce qui revient à douter de son honnêteté ou de son équilibre mental. Aussi, en religion, chacun peut-il prétendre faire autorité parce qu'en dernier recours, c'est toujours l'expérience intime qui arbitre la "vérité».

La tradition, en cela, n'annule aucunement l'expérience. Au contraire, elle convoque des milliers de croyants des générations passées qui ont tenu à léguer ce trésor à leurs descendants et elle mène toujours, en dernier recours, à une expérience originaire attestée par des témoins privilégiés. Le débat religieux se présente ainsi comme le faire-valoir d'expériences qui répondent toutes, chacune à leur façon, à une histoire de salut. L'expérience religieuse assumée pose ainsi que, sans elle, la vie serait impossible. Si bien qu'en dernière analyse, chacun est forcément l'incroyant de celui qui croit autrement.

Quels qu'ils soient, en ostensoir ou en sautoir, en éblouissantes chorégraphies rituelles ou en gestes furtifs, les signes religieux - comme leur contestation - renvoient à la quête d'un sens qui se profile, souvent indéfinissable, à l'horizon des terres traversées par chacun. Généralement, on clamera ce sens d'autant plus fortement que l'évidence en est fragile. Le seul fait de le faire venir au langage, dès lors, lui donne puissance parce que cet acte, déjà, le rend vraisemblable et en permet le partage. Dire le sens, c'est accéder à une vie sociale. C'est se rendre disponible à la reconnaissance des autres. C'est se positionner dans l'ordre qui dissout le désordre, dans le nomos susceptible de vaincre le chaos. C'est contrer l'arbitraire pour rendre la vie possible. 
Derrière leur matérialité dérisoire, les signes religieux rappellent que les humains ne peuvent échapper au risque qui consiste à gérer leur vie en misant sur de la représentation. Sous le mode de la domination quand leurs traditions s'imposent, sous celui de la concurrence quand leurs signifiants sont mis en libre commerce, les institutions religieuses tentent d'encadrer cette entreprise et prétendent la guider au sein des cultures. Les incroyants n'y échappent pas plus que les autres, dans la mesure où ils sont mis en demeure, eux aussi, de faire valoir leur rapport au sens et de mettre à nu leur expérience. La difficulté du débat consiste alors à relativiser cette représentation du monde qui permet de vivre, pour se rendre disponible à l'autre. Pour le croyant - incroyant de celui qui croit autrement -, c'est un non-sens: c'est lui demander de renoncer à ce qui le fait vivre.

On est en présence ici de systèmes de convictions. Et les adeptes de n'importe quel système de conviction, affirmative ou négative d'une expérience donnée, ne sont pas ultimement intéressés à s'engager dans le dialogue 5 . Ils veulent que leurs croyances prédominent. Cela paraît normal quand on considère qu'elles représentent, pour eux, la vie par rapport à la mort. Quand il soulève des questions religieuses, le débat public devient structurellement aporétique. On ne peut sortir de l'impasse en débattant sans fin de qui a tort ou raison. Il faut trouver autre chose.

Certaines exigences s'avèrent dès lors incontournables pour que la communication devienne possible. Tout d'abord, chaque interlocuteur doit prendre en considération le fait que la conviction de l'autre, autant que la sienne, est l'expression d'une quête. Derrière les certitudes mises de l'avant se terre toujours la fragilité commune des humains. Or, l'attention à la quête, précisément, permet d'accéder à cetre réalité et, éventuellement, de la partager. Pour peu que chacun reste honnête avec lui-même, elle permet de défricher un terrain commun. Le langage peut alors jouer son rôle: permettre l'entente entre des humains soumis à des expériences différentes. Nous appelons cette exigence une éthique de la posture.

Cela ne veut pas dire qu'il faille renoncer à ses convictions. En toute situation, la condition élémentaire d'une éthique de la communication est que les convictions des interlocuteurs soient d'abord mises au clair. Cependant, la conviction est comme une plante: elle se nourrit du terreau d'où elle germe pour s'exposer à la lumière. Dans une éthique de la posture,

$$
++
$$

5. David MENDELSOHN, «Logique laique er volonté divine: La croisée des chemins", Les Cahrers du 27 jün, vol. 3, $n^{\circ} 2$, automne-hiver 2007, p. 40-43. 
il s'agit très précisément d'explorer ce terreau, de convoquer la fragilité même de l'être à s'exposer. Cela suppose bien sûr une ascèse. La conviviance entre des structures de conviction différentes appelle non seulement le respect de l'autre, l'écoute qui lui fait hospitalité, mais aussi la mise à nu de chacun des interlocuteurs, qui se font ainsi réciproquement hospitalité.

Sinon, il est bien évident que chacun ne peut que réaffirmer sans cesse les clauses prétendues intouchables de ses conventions sociales. Or, il arrive qu'il en soit ainsi, aujourd'hui, non seulement de ceux dont l'expérience religieuse est fragilisée par la modernité - héritiers des traditions endogènes ou importateurs de traditions exogènes peu importe -, mais aussi de ceux qui défendent le courant central des régulations sociales dites "modernes" et affirment, pour cela, leur refus des autres. Quand on déclare, par exemple, qu'il existe un intouchable dans la culture québécoise et qu'il concerne l'égalité entre les hommes et les femmes, on procède explicitement à une double production religieuse: l'énoncé d'un espace sacré et celui d'un mythe. L'«intouchable», c'est la définition même du sacré. Le mythe consiste à faire comme si l'égalité, dont personne par ailleurs ne saurait contester l'idéal, concernait une réalité déjà là dont on détiendrait la garantie. Certes, compte tenu de sa fragilité, il est de bonne guerre de sacraliser une cause comme celle de l'égalité. Mais cela permet aussi de détourner le regard des dynamiques d'inégalité qui non seulement perdurent, mais travaillent à ciel ouvert les sociétés occidentales. Pourtant, si l'on y porte quelque peu attention, il faut bien poser l'hypothèse que la régulation marchande de la sexualité, voie royale d'accès au bonheur, promeut des stéréotypes du masculin et du féminin plus forts que jamais. La sexualisation précoce des petites filles dénoncée par les sexologues et les "burkas de chair" de leurs aînées, évoquées par Nelly Arcan ${ }^{6}$, ont peu à envier aux voiles des femmes du désert comme violence faite aux femmes. Les simulacres phalliques érigés par leurs partenaires dans leur démonstration de puissance, ou quand ils sont en mal de séduction, relèvent aussi de cette dynamique. Pour s'en convaincre, il suffit d'observer les rituels des téléréalités ${ }^{7}$. Certes, on peut se satisfaire d'une culture de ce type. Mais saisie par un regard distancié, ce qui est relativement facile pour un étranger, elle présente aussi des violences scandaleuses.

Assumer le pluralisme consiste ainsi à intégrer quelque chose du regard de l'autre.

$$
+4
$$

6. Nelly ARCAN, $A$ ciel ouvert, Paris, Seuil, 2007. Voir aussi son article "La disparition des femmes», LAcrualite, 15 septembre 2007, p. 105-106.

7. Voir Jean-Pierre DeSAUl.NIERS, "Lofi Story, ou la dernière scène ”, Le Devoir, 17 ocrobre 2003, p. A10. 


\section{DEUXIÈME DÉFI : ASSUMER LA MODERNITÉ}

Dans les paragraphes précédents, nous avons appelé sens la signification globale qu'un sujet, individuel ou collectif, peut donner à ses pratiques, à son discours ou à sa situation. Nous appelons religion, dans cette foulée, les dispositifs culturels qui se mettent en place pour garantir ce sens et en assurer la régulation. Il s'agit alors, en général, d'attester de l'authenticité de l'expérience qui en est la source, de même que de sa capacité à produire le bien pour ceux qui l'acceptent. Les signifiants ainsi retenus, qui forment l'ossature d'un discours religieux donné, de même que les représentations du monde dont ils dépendent, deviennent alors pour ceux qui les adoptent autant de guides de survie dans la traversée de l'existence. La religion joue ainsi un rôle de normatisation (qu'il ne faut pas confondre avec une normalisation) par lequel l'espace du sens - jusqu'au cosmos sacré évoqué par Peter Berger ${ }^{8}$ - se distingue du non-sens. Elle ne fait pas disparaitre le chaos, mais elle élabore, en son sein même, des espaces symboliques qui rendent possible la vie des individus et des groupes.

Il est bien entendu que pour le croyant, ces espaces symboliques sont incontournables. Ils ne peuvent représenter qu'un don de l'Autre, un Autre dont il se figure la réalité dans l'axe même de la cohérence du monde qu'il en reçoit. Pour l'incroyant, à l'inverse, ce don est illusoire. Mais il peut très bien, du même coup, chercher ailleurs le fondement du sens: dans d'autres traditions et religiosités, ou encore dans la reconnaissance d'une détermination inhérente à la matière même du cosmos, voire dans l'absolutisation de ses valeurs devenues intouchables. La posture analytique promue plus haut ne conteste pas la logique de ces positions. Elle pose simplement que s'il y a illusion, c'est une illusion nécessaire à la vie, traduction d'une quête qui mérite d'être respectée parce qu'elle témoigne de l'humain.

Au XXe siècle, une profonde rupture s'est produite dans les sociétés occidentales quant à leur rapport au sens. Depuis plus d'un millénaire, celuici était porté par des institutions religieuses capables de l'encadrer et de le garantir. Certes, au rythme du développement d'une modernité opposant aux visions religieuses du monde une approche technique et rationnelle, fondée sur l'efficacité plutôt que sur l'autorité, on a pu facilement observer l'effritement de cet encadrement au cours des derniers siècles. Mais le milieu du XXe siècle a marqué à cet égard une sorte de point de non-retour; le statut

$$
+4
$$

8. Peter BERGeR, La religzon dans la conscience modeme, Paris, Centurion, 1971. 
du religieux a basculé. Jusque-là, il garantissait l'entente à l'intérieur des communautés humaines, en regard d'un sens partagé par leurs membres et à l'encontre des autres, ceux-ci devenant de ce fait étrangers, étranges, infidèles et dangereux pour la cohésion interne. Le statut du religieux est alors devenu le support de quêtes de sens personnelles pour des individus qui se veulent autonomes et cherchent pour eux-mêmes, sur un marché ouvert de signifiants et d'appartenances, ce qui peut les satisfaire.

L'affirmation de la modernité a ainsi pu disqualifier les formes traditionnelles de la culture, notamment les cléricales jugées routinières et intrusives, pour leur opposer la liberté enthousiaste des individus en quête de réalisation personnelle et d'émancipation. Remarquons que cette transformation ne signe en rien la disparition du religieux. Elle engage plutôt une mutation de sa fonction sociale. L'un des symptômes de cette mutation est l'émergence de toutes sortes de phénomènes paradoxaux dans les dernières décennies du XXe siècle: nouveaux mouvements religieux, quêtes de spiritualité au sein de traditions exogènes (notamment orientales, amérindiennes et nouvelâgistes), effervescences religieuses en marge des institutions, "réveils" de toutes sortes, etc.

La rupture d'avec les traditions devient ainsi fondatrice. Loin d'être indifférente au religieux, la culture nouvelle en fait au contraire un objet de débat de plus en plus virulent. Les signes religieux se multiplient, alors même que les signes traditionnels s'effacent. Mais ce religieux qui agresse l'idéal séculier et fragilise la laïcité des institutions publiques n'est plus du tout de la même nature que celui d'autrefois. Il ne garantit plus une identité collective en lutte contre d'autres identités collectives, mais, conséquence directe de la liberté et de sa régulation marchande, il appartient d'abord aux individus en quête de satisfaction. Il marque alors non plus des ressemblances, mais des différences. De garantie de l'entente quant au sens qu'il représentait, il est devenu le support de quêtes personnelles pour des individus qui sont en droit de faire valoir, sur la place publique et non seulement dans les cryptes du privé, la spécificité de leur identité et de leur histoire.

Le religieux n'a jamais disparu des sociétés occidentales. Bien au contraire. Il devient cependant l'objet d'une régulation complètement étrangère à celle qu'il a jusque-là connue. Les croyances, rites et appartenances autrefois gérés par des institutions capables de négocier, sinon d'imposer leur reconnaissance publique, sont désormais objets d'une régulation marchande, fondée sur l'offre de satisfactions aux quêtes de sens personnelles. On n'a, s'il faut s'en convaincre, qu'à consulter les récits de conversion, quel qu'en soit 
l'objet: le sujet y raconte généralement comment, au terme d'une longue pérégrination, il a enfin trouvé la réponse à ses questions?.

Cette régulation marchande des biens de salut a des conséquences majeures sur les formations religieuses, traditionnelles ou non. Tout d'abord, elle en provoque l'éclatement. La logique de marché, qui consiste à offrir des produits susceptibles de satisfaire des "besoins", tend en effet à démultiplier ses offres et à valoriser les différences marginales pour rejoindre le plus grand nombre de clients possible. Elle favorise ainsi, dans le champ religieux comme dans celui des autres consommations, un rapport identitaire entre le client et le produit consommé.

En prolongement immédiat de cette première conséquence, on assiste alors à la désinstitutionnalisation des identités religieuses. Pourquoi, en effet, un sujet "rationnel" s'en référerait-il aux contraintes et rigidités d'une tradition s'il peut trouver satisfaction en choisissant ce qui lui convient sur un marché ouvert? Il parcourt alors les étalages de sens offerts au tout venant pour puiser, là où il les trouve et en exerçant son sens critique, les produits qui contribueront à la cohérence non pas doctrinale, mais proprement affective et personnelle, de son univers de sens.

On se leurrerait en concluant à un affaiblissement de la conviction. Bien au contraire - et c'est là que le bât blesse quand on défend la laïcité des institutions publiques -, on trouve en fin de parcours une radicalisation de la conviction et de la revendication de son droit à s'exprimer sur la place publique. Quand un individu découvre enfin - comme en témoignent les récits de conversion - la solution à sa quête, tout d'abord, il y tient... C'est là, pour lui, question de survie. Plus elle sera contestée par d'autres, plus il s'y accrochera - ce qu'ont démontré d'ailleurs depuis longtemps les études de psychologie sociale ${ }^{10}$. Ensuite, il entend la partager. Le fait qu'il puisse le faire témoigne de sa vraisemblance: si d'autres sont d'accord avec moi, je ne puis être complètement dans le champ... Se forment ainsi une myriade de cellules croyantes qui chercheront à leur tour à multiplier les alliances et radicaliseront leur discours d'autant plus que le chaos leur semble dominer autour d'elles et qu'elles restent foncièrement fragiles dans leurs propres assises. Dès lors, ces croyants deviennent des groupes de pression qui feront valoir leurs positions sur la place publique, à

$$
+4
$$

9. Ceci est particulièrement explicite dans les conversions des Occidentaux à l'ıslam. Voir, par exemple, Lisbech RoCHER et Fatıma Cherquaou, D'une foi a l'autre. Les conversions à l'tslam en Occident, Paris, Seuil, 1986.

10. Voir, entre autres, les recherches de Leon FESTINGER et coll., L'échec d'une prophéte. Psychologie sociale diun groupe de fideles qui prédisaient la fin du monde, Parıs, Presses universitaires de France, 1993 [1956]. 
l'encontre même des principes laïcs qui fondent cette place et permettent, par leur libéralisme, de s'y faire entendre.

Le Québec n'a pratiquement pas connu, jusqu'ici, le fondamentalisme, pourtant bien vivant dans d'autres pays où dominent les régulations de marché. La persistance d'un encadrement catholique l'en a peut-être protégé! Il faut bien voir à ce propos que celui-ci, depuis la Conquête, a essentiellement diffusé une idéologie de la survivance, dans un contexte de lutte identitaire face à un dominant d'une autre culture. Certes, il a connu également des accents missionnaires et conquérants. Mais ces derniers étaient-ils si différents de ceux du reste de l'Occident? Jusqu'à quel point ont-ils représenté, dans leur avatar catholique, la simple traduction du mythe commun de la mission civilisatrice de l'Occident qui, lui, semble encore loin d'avoir baissé les armes?

Le fondamentalisme est aussi un sous-produit de la logique de marché. Offerts en objets de consommation "satisfaisante", les biens religieux deviennent comme tous les autres biens de consommation, addictifs: il en faut toujours davantage pour obtenir une satisfaction toujours menacée. Comme sur les autres marchés, le client idéal est celui qui augmente les doses et rapproche les prises. Quitte à y risquer sa propre vie. La montée en force des groupes de conviction, à partir du moment où elle présente des quantités appréciables d'électeurs potentiels, ne laissera pas non plus indifférents ceux qui ont besoin de consolider leur pouvoir sur la place publique. Ces électeurs deviendront des alliés potentiels précieux dans les jeux politiques. Ils donneront à leurs partenaires des assises, tant électorales qu'idéologiques, pour affirmer leur légitimité et y trouveront pour euxmêmes un pouvoir qu'ils ne pourraient conquérir autrement. La laïcité des institutions publiques risque par conséquent de devenir toute symbolique, malgré les principes qui semblent la garantir.

Assumer la modernité, c'est se rendre compte que celle-ci est, tout autant que la tradition, productrice de forces religieuses.

Dans sa conception des institutions publiques américaines, conception qui prône comme on le sait la séparation radicale des Églises et de l'État, Thomas Jefferson a institué, en bon libéral, non pas la tolérance, mais bien la liberté religieuse. La première dominait alors la pensée des Lumières qu'il connaissait bien. Il ne l'a pas fait contre les confessions religieuses déjà en concurrence dans les États naissants, mais bien en pensant 
que cette concurrence, comme la théorie libérale lui permettait de l'envisager, favoriserait leur développement. Pari gagné si on considère que les États-Unis sont devenus l'un des pays les plus religieux du monde. Pragmatique, Jefferson croyait aussi tenir là la clé de la paix sociale, ce à quoi la tolérance n'arrivait pas. Et pour bien s'assurer que les fragiles équilibres de marché ne seraient pas perturbés par les intrusions de l'État, il a institué entre celui-ci et les Églises un "mur de séparation" qui caractérise la laïcité américaine.

Cette pragmatique de la liberté religieuse est cependant aujourd'hui remise en question. Sous l'emprise des régulations de marché, la liberté donne de l'espace à la radicalisation des convictions, comme elle en donne aux prédateurs en d'autres domaines. Entendant tenir les positions les plus fortes, les acteurs politiques cherchent l'appui des religieux pour légitimer leurs politiques, qui deviennent littéralement des luttes du bien contre le mal, c'est-à-dire des politiques proprement religieuses.

Le Québec contemporain est-il à l'abri d'un tel programme? Nous ne saurions en décider. La question mérite pourtant d'être posée. 